\title{
Die Periodik der Lebenserscheinungen beim Menschen.
}

\author{
Von \\ Professor Dr. Theodor Brugsch, \\ Oberarzt der II. med. Klinik der Charité Berlin.
}

Ueberall in der Natur begegnen wir der Periodizität, dem Kreislaufe der Natur. Werden und Vergehen, Jahreszeiten, Tag und Nacht, Ebbe und Flut in ihrem regelmäßigen Wechsel sind solche periodischen Erscheinungen unserer Welt, die sich dem unbefangenen Beobachter aufdrängen, und da der Mensch als Bewohner unseres Planeten Erde ein Teil dieses Planeten ist und sich mit der Umwelt ins Gleichgewicht setzen muß, liegt der Gedanke wohl nahe, daß die Periodizität der Natur auch die Periodizität gewisser Lebenserscheinungen unseres Organismus bewirke. Jedes Geschehen ist ein zeitlich-räumliches und es ist die Frage der Untersuchung wohl wert, inwieweit in unserm Organismus überhaupt periodische Lebenserscheinungen auftreten und ob diese Erscheinungen sich als abhängig von Bedingungen außerhalb unseres Organismus erweisen.

1. Der menschliche Organismus als physiologisches System vom energetischen Standpunkte aus und oscillatorische Erscheinungen im allgemeinen.

Wir fassen die Konstitution eines menschlichen Individuums als ein vitales System von Massenpunkten auf, deren raum-zeitliche Lage sich in einem Gleichgewichtszustande unter sich und mit der Außenwelt befindet. Wenn wir die inneren Zusammenhänge als $I$, die äuBeren Zusammenhänge als $\mathrm{A}$ bezeichnen, so kann man 
diesen Gleichgewichtszustand durch ein Formelsymbol etwa so ausdrücken:

$$
\mathrm{C}=\mathrm{I} \rightleftarrows \mathrm{O} \rightleftarrows \mathrm{A} \text {. }
$$

wobei $\mathrm{O}$ das Symbol für die körperliche Organisation in struktureller Beziehung bedeutet (kürzer ausgedrückt für die Organisation).

Dieser Formulierung liegt (konform mit $\mathrm{C}$ oh e $\mathrm{n}-\mathrm{Kys} \mathrm{p}$ er) eine mechanische Auffassung im $\mathrm{Hertz}$ schen Sinne zugrunde und läßt sich auch auf jede energetische Lebensauffassung, die nicht nur auf der mechanischen Theorie der Bewegung basiert erweitern. Läßt sich Masse und Energie identifizieren und alles Geschehen nicht mehr als mechanische Bewegung auffassen, sondern nur noch elektrodynamisch, so wird unsere Betrachtungsweise sich auch diesem allgemeineren Standpunkte unterordnen können; für unsere Fragestellung aber besitzt eine Formulierung im $\mathrm{Hert} z$ schen Sinne den Vorzug eines gewissermaßen fixierten Koordinatensystems.

Unser Organismus strebt einem Gleichgewichtszustande entgegen, das wären unmittelbar die Folgerungen, die wir aus dieser Definition ziehen müssen. Man kann rein praktisch, ohne Voreingenommenheit, diese Folgerungen auf ihre Richtigkeit prüfen und man wird eine Bestätigung für den ausgewachsenen Organismus finden. Der menschliche Organismus gleicht sich im allgemeinen von einem Tag auf den andern. Es soll dabei das psychische Verhalten ganz auBer acht gelassen werden und nur das somatische Verhalten berücksichtigt werden. Stellt man einen ausgewachsenen Menschen, sofern er annähernd unter gleichen äußeren Bedingungen bleibt, auf die Wage, so findet man, wenn man 24stündig unter gleichen äußeren Bedingungen mißt, Gewichtskonstanz. Mißt man allerdings stündlich, so zeigen sich Schwankungen um einen Mittelwert, die wir einmal Oszillationen nennen wollen. Diese Oszillationen bekräftigen nur das, was wir als Streben nach einem Gleichgewichtszustand ausgedrückt haben. Aehnliche Schwankungen um eine Mittellage beobachtet man bei der Feststellung der Körpertemperatur bei Messung in einer Körperhöhle; stellt man die Blutdruckkurve fest, so ergeht es ähnlich. Ueberall das Streben nach funktioneller und struktureller Gleichgewichtslage unter den Erscheinungen von Oszillationen. $\mathrm{D}$ a $\mathrm{m}$ it geraten wir bereits auf das Gebiet der periodischen $S c h w a n k u n g e n$ bei einem Proze $B$ den 
wir im ganzengenommen als stationären Pro$z$ e $B$ bezeichnen müssen.

Auf den Organismus wirkt mit der Atmung der Sauerstoff der Luft ein, in den Organismus fließt ein Strom von Nahrung ein, aus dem Organismus wird $\mathrm{CO}_{2}$ und $\mathrm{H}_{2} \mathrm{O}$ abgedunstet, fließt Wärme $a b$, fließen Exkrete und Sekrete ab, wobei die Ausscheidungen energetisch qualitativ durchaus anderer Natur sind als die Einnahmen. Aber gerade diese Bedingungen, unter denen der stationäre Zustand erhalten bleiben kann, erfüllt die $\mathrm{H}$ e $\mathrm{r}$ i n $g$ sche Theorie der Dissimilation-Assimilation, die man am einfachsten durch die Formulierung

$$
\frac{\mathrm{D}}{\mathrm{A}}=1
$$

energetisch zum Ausdruck bringen kann. Die eintretende Dissimilation wird durch die Assimilation wieder wettgemacht; dieser Prozeß verläuft im einzelnen als Carnotscher Kreisprozeß, und wenn man den Organismus als Ganzes nimmt, als eine große Summe von Kreisprozessen, die allerdings zusammengehalten werden müssen, und das ist nicht anders möglich, durch die periodischen großen Bewegungen (Kreislauf, Atmung), von denen wir im nächsten Abschnitt abzuhandeln haben.

Betrachten wir noch einmal für sich den Dissimilation-Assimilationsprozeß, so müssen sich im einzelnen Oszillationen ergeben, weil jeweils die Dissimilation zeitlich der Assimilation vorangeht, die Steuerung dabei durch einen automatischen Regulationsfaktor gegeben sein muß, der in den durch die Dissimilation sich ergebenden Abbaustoffen zu suchen ist. Um das plausibler zu machen mag auf einen von W. Ost w ald herangezogenen Vergleich verwiesen werden, nämlich die brennende Kerze. Diese ist unmittelbar nach dem Anzünden der Flamme klein. Hierdurch ist sie der Oberfläche des Wachses besonders nahe, schmilzt Wachs ein und brennt dadurch empor; dadurch entfernt sie sich aber wieder von der Wachsoberfläche, was zur Verminderung der Flamme und zum Niedersteigen führt und so fort. Für die ungenaue kurze Beobachtungszeit erscheint die Flamme gleichbleibend, bei näherer Beobachtung ergibt sich aber deutlich die Periodik der Flamme, die wir eine automatische regulierte Oszillation nennen wollen. 
Soweit also in unserem Organismus Kreisprozesse im Sinne von $\frac{\mathrm{D}}{\mathrm{A}}=1 \mathrm{im}$ einzelnen zustande kommen, müssen wir mit Oszillationen rechnen und wenn wir den Komplex von Kreisprozessen, zusammengehalten durch große periodische Bewegungen, zusammennehmen, so erscheinen naturgemäß die Oszillationen deutlicher im Ausschlag. Ohne Oszillationen ist der stationäre Prozeß nicht aufrecht erhaltbar, sind Kreisprozesse nicht denkbar und da energetisch das Leben ein Dissimilations-AssimilationsprozeB ist, so ist die oszillatorische Periodenbildung, wie sie uns im großen durch funktionelle und strukturelle Schwankungen des menschlichen Soma im stationären Gleichgewicht entgegentritt, ein Etwas, was schlechterdings nicht aus dem Leben fortzudenken ist: nichts von der Außenwelt aufgedrungenes, sondern dem lebenden System generell immanentes, d. h. innere Bedingung jeder lebenden Substanz in der Wechselwirkung mit der Außenwelt.

\section{Die periodischen Bewegungen.}

Die Kreisprozesse mit ihren Oszillationen im einzelnen werden durch große Bewegungsprozesse zusammengehalten, d. h. die Bewegungen des Kreislaufes, die Atembewegungen, die Muskelbewegungen und endlich die Darmbewegungen. Der stationäre Zustand des Organismus oder seine Gleichgewichtslage wird nicht etwa durch diese Bewegungen gestört, sondern geradezu bedingt, so wie ein drehender Kreisel seine Gleichgewichtslage durch die Drehung beibehält. Können wir die Oszillationen des $\frac{\mathrm{D}}{\mathrm{A}}$-Prozesses als etwas der lebenden Materie immanentes ansehen, so ist die Frage des Zusammenhanges dieser periodischen Bewegungen oder Schwankungen mit Faktoren der Außenwelt viel näherliegend. Unvoreingenommen wird ein jeder Zusammenhänge zwischen diesen periodischen Bewegungen und der Umwelt zugestehen müssen. Steigen wir eine Treppe hinauf, so steigt Puls und Atmung an, befinden wir uns im Schlafe, so nimmt deren Erregung $a b$. Wechseln wir den Ort, so steigen beide mit abnehmendem Barometerdrucke an, ändern den Rhythmus bei veränderter Außentemperatur usw. Der Rhythmus der Darmbewegungen wird endlich durch die Nahrungsaufnahmen durchaus beeinfluBt. Aber über derartige jedern Laien leicht zugängliche und 
jedem Arzte geläufige Beziehungen hinaus, zeigt sich für uns ein tieferes Interesse für die Frage, ob sich die Periodik dieser Bewegungen schon in ihrer Anlage als abhängig von Faktoren der AuBenwelt zeigt, vielleicht sogar schon Zusammenhänge aufweist mit der pulsierenden Vakuole einzelliger Tiere?

Wir müssen naturgemäß die Frage der Periodik der Bewegunger: in unserm Organismus einheitlich zu beantworten suchen, müssen darum auch eine Vorfrage erledigen, ob nämlich zwischen Kreislaufs-, Atmungs-, Muskel- und Darmbewegungen Zusammenhänge existieren, die eine einheitliche Beurteilung der Bewegungsperiodik gestatten. Solche Zusammenhänge existieren: der unter normalem Verhältnis konstante Wert von 3,5-4 als Verhältniszahl von Pulsund Atmungsfrequenz spricht ohne weiteres dafür. In pathologischen Fällen wird dieses Verhältnis durchbrochen, wenn das Herz insuffizient ist oder die respirierende Lungenoberfläche verkleinert ist oder nervöse Regulationsstörungen vorhanden sind. Dabei darf man dem Herzrhythmus die Rolle der primären Periodik zuschreiben, da ein solcher schon fötal angelegt ist, die Atmungsrhythmik sich erst später postfötal einstellt. Aber auch zwischen der Muskelbewegungsrhythmik und der Herzrhythmik muß ein solcher Zusammenhang angenommen werden, da ja Muskelbewegungen die Herzperiodik ändern; unbefangen können wir aus der frühen Anlage des Herzrhythmus im fötalen Leben gegenüber der späteren Ausbildung geordneter Muskelbewegung dem Herzrhythmus die Ueberordnung in zeitlicher und - aus der zentralen Anlage heraus - auch in räumlicher Beziehung zusprechen. Am schwierigsten ist die Frage der Rhythmik der Darmbewegungen $z u$ bewerten. Die Leertätigkeit des MagenDarmkanals ist periodisch, die Periodik stellt sich auf Nahrungsaufnahme neu ein; naturgemäß ist Herzrhythmik die Vorbedingung für jede Darmperiodik, aber es soll doch die Frage geprüft werden, ob ein noch engeres, gewissermaßen periodisch gebundenes $\mathrm{Ab}$ hängigkeitsverhältnis besteht. Diese Frage ist schwer zu beantworten, da es an Beweisen fehlt; wahrscheinlich ist aber auch hier die Abhängigkeit der Periodenbildung von der Herzrhythmik, nur muß erst einmal dieser Frage noch besondere Aufmerksamkeit geschenkt werden. Wir wollen aber für unsere Studie eine solche Abhängigkeit einmal annehmen, dabei aber auch wieder dem Herzen das Primordiat der Periodik zuerkennen. Dann hätten wird das Recht zu dem Ausspruche: jegliche Bewegungsrhyt h- 
mik in unserm Organismus ist der Herzrhythmik untergeordnet.

Die Bewegungsperiodik wollen wir nunmehr energetisch fassen und uns speziell mit der des Herzens beschäftigen. Wir gehen wieder von dem stationären oder Gleichgewichtszustande des Organismus aus und definieren jetzt mit $\mathrm{Z}$ wa a $\mathrm{r}$ e maker den Gleichgewichtszustand des Organismus als einen Gleichgewichtszustand koexistierender heterogener Phasen, wobei das Gleichgewicht durch Druck, Temperatur, thermodynamisches Potential und die Mischungsverhältnisse der Komponenten, sowie durch die in den Phasen vorhandenen kapillären Verhältnisse bestimmt wird. Es ist dabei der physiologische Organismus als ein (verwickeltes) System von heterogenen Phasen betrachtet, so wie etwa die Zellenlehre den Organismus als ein System koexistierender Zellen auffaßt, nur daß die Phase hier nicht Zelle bedeutet, sondern (nach $\mathrm{Gib}$ b s) die stofflichen Aggregatzustände; das Problem wird damit physikalisch-chemisch gestellt. Tritt nun an irgendeiner Stelle des Organismus eine autochthone Periodik, z. B. eine rhythmische Muskelbewegung auf, so ist mit dem jedesmaligen Auftritt des Bewegungsvorganges eine Gleichgewichtsstörung verbunden, aber nicht in dem Sinne, daß die Bewegung die Störung des Gleichgewichts hervorruft, sondern daß eine Aenderung des Gleichgewichts die Bewegung hervorruft. Betrachten wir die Verhältnisse bei der Herzperiodik im einzelnen. Der Reizbildungs- und Reizleitungsapparat stellt hier den Phasenkomplex $R$, der Muskelapparat einen Phasenkomplex $M$, und das Blut den Phasenkomplex $S$ dar. Eine Gleichgewichtsstörung muß durch Ausgleich dieser Störung den ersten Herzschlag bedingen. Am herausgeschnittenen und mit Ringerscher Lösung durchspülten Herzen läßt sich studieren, wie die Herzrhythmik ausgelöst wird durch einen Ausgleich zwischen den Phasenkomplexen $S$ und $R, R$ und $M$ unter der Ausgleichsbedingung zwischen $M$ und $S$. Ein dreifacher prinzipieller Phasenkomplexausgleich muß also zum Zustandekommen der Herzperiodik vorhanden sein. Betrachten wir zunächst den Ausgleich zwischen dem Phasenkomplex S und R. Das Blut stellt einen vom Bau des Reizteilungssystems völlig differenten Phasenkomplex dar, wobei wir aber der Einfachheit wegen beim Blut nur das Verhalten der molekulardispersen Stoffe in der flüssigen Phase des Blutserums verfolgen wollen. Man kann ja mit Ringerscher Lösung dasselbe Ergebnis der Periodik am herausgeschnittenen 
Herzen erzielen, was das Blut am uneröffneten Organismus vollbringt. Bei der Ringerlösung (bzw. Ringer-Lockeschen) erhält man bei der Herzdurchströmung die günstigsten Resultate, wenn man auf $1000 \mathrm{~g}$ Wasser $9-10 \mathrm{~g} \mathrm{NaCl}, 0,2 \mathrm{~g} \mathrm{KCl}, 0,2 \mathrm{~g} \mathrm{CaCl}_{2}, 0,1 \mathrm{~g} \mathrm{NaHCO}_{3}$ und 1,0 $\mathrm{g}$ Traubenzucker zusetzt; dabei kommen auf 100 Molen $\mathrm{NaCl} 2 \mathrm{Molen} \mathrm{KCl}$ und $1 \mathrm{Mol} \mathrm{CaCl}_{2}$. In demselben äquimolekularen Verhältnis enthält aber durch die ganze Säugetierreihe hindurch (z. B. Mensch, Rind, Schaf, Ziege, Pferd, Schwein, Katze) das Blutserum diese Stoffe, ja darüber hinaus enthält auch das Meerwasser diese Stoffe in demselben äquimolekularen Verhältnis. So wird die flüssige Phase von S, die in Ausgleich tritt zum Phasenkomplex R gewissermaßen zur Durchspülungsflüssigkeit vom Charakter des Meerwassers. Betrachten wir nunmehr die Verhältnisse bei dem Phasenkomplex $\mathrm{R}$, dessen anatomische Struktur der quergestreiften Muskulatur des Herzens gleicht. Leider besitzen wir von der Muskulatur noch keine Vorstellung über die molekulardispersen Stoffe seiner flüssigen Phase. Nur soviel wissen wir, daß während das Blutserum reich an Na-ion, die Muskulatur arm daran ist, während das Serum arm an Kali-ion ist, der Herzmuskel reich daran ist, im Muskel prävaliert Schwefelsäure und vor allem Phosphorsäure, im Serum die Salzsäure, mit anderen Worten: Serum und Muskulatur sind in der Zusammensetzung der molekulardispersen Kristalloide als unterschieden anzunehmen und da zwischen $S$ und $R$ eine semipermeable Wand als Trennungsfläche vorhanden sein muB, an der nach der $G$ i b b s'schen Phasenregel der Ausgleich sich vollzieht, so ist wohl die Annahme berechtigt, daß durch Uebertritt von Ionen (und zwar Na-ionen) aus dem System $S$ nach $R$ die Periodik ausgelöst wird. Es soll hier nicht auf die Frage näher eingegangen werden, inwieweit das Verhältnis anderer Ionen auf die Permeabilität der Wand Einfluß hat, inwieweit eine Diffusion von $R$ nach $S$ stattfindet: hier kommt es ja nur auf die Darstellung des Problems im allgemeinen an und da genügt es für uns, daß eine Ionenwanderung aus einem Milieu, das dem Meerwasser gleicht, die Bewegungsperiodik auslöst; nunmehr vollzieht sich die zweite Ausgleichsreaktion in den Phasenkomplexen $R$ und $M$. Wir nennen diese Ausgleichsreaktion eine Reizleitung und können über das Geschehen insofern etwas aussagen, als ein elektrischer meBbarer Strom (Aktionsstrom) uns die Annahme nahelegt, daB es sich um eine Ionenwanderung handelt. Schwieriger ist schon die Frage zu erledigen, 
wieso innerhalb der Phasengruppe $M$ durch den Anstoß einer Ionenverschiebung die Zuckung zustande kommt. Man muß sich vorstellen, daB der ruhende Muskel sich im falschen Gleichgewicht befindet und daß durch die Ionenverschiebung die kontraktile Substanz in die richtige Gleichgewichtslage kommt. Bei diesem Uebergange von der falschen Gleichgewichtslage in die richtige kommt es dann durch Verbrauch von potentieller Energie zu Diffusionsströmen zwischen Sarkoplasma und kontraktiler Substanz, die ein Zurückgehen in die frühere falsche Gleichgewichtslage bedingen. So wird auf das System der kontraktilen Substanz der Ursprungsreiz durch lonenwanderung vom Reizzentrum aus vermittelt, und im Reizzentrum die Ionenwanderung aus dem Blutserum in das Zentrum zum Anlaß der Reizbildung. Das gleiche Prinzip aber hat auch statt in der übrigen Muskulatur: bei der glatten Muskulatur des Darmes mit der periodischen Pendelung läßt sich eine gleiche Einrichtung wie beim Herzen supponiern, d. h. durch Ionenwanderung auf Plexus als Reizursprungsgebiet die Auslösung des Reizes, durch Ionenwanderung die Auslösung der Kontraktion. Schwieriger liegen schon die Verhältnisse im quergestreiften willkürlich bewegten Muskel; bedeutet aber der Reiz im Nerven eine lonenwanderung, so tritt auch hier wieder die Zuckung gleichsinnig ausgelöst auf wie beim Herzmuskel. Schwer zu beantworten, ist nur die Frage, inwieweit wieder das Serum (die Phasengruppe S) an der ideomotorischen Reizbildung beteiligt ist. Unbeteiligt ist es jedenfalls nicht, sonst würden Ermüdungsstoffe des Blutes wie man sie durch Blutübertragung vom ermüdeten Tiere auf das unermüdete nachweisen kann, fehlen. Kompliziert liegen auch die Verhältnisse bei der Atmung, indessen ohne weiteres eindeutig, vergleichbar mit den Verhältnissen beim Herzen und der glatten Muskulatur, nur mit dem Unterschiede noch, daB, wie wir jetzt wissen, Frequenz und Tiefe der Atemzüge in erster Linie durch die $\mathrm{H}$-Ionenkonzentration des Blutes bestimmt wird. Alles in allem: der ursprünglichsten und übergeordneten Bewegungsperiodik, der Herzperiodik, liegt ein System zugrunde, das mehr oder minder ähnlich auch bei anderer Bewegungsperiodik (Atmungs-, Muskel-, Darmperiodik) sich findet und das im Beginne zur Auslösung gebracht wird durch eine Ionenverschiebung vom Blutserum nach dem Orte der Reizbildung, wobei biologisch die elementare Salzzusammensetzung der Blutsera des Menschen und vieler Wirbeltiere auf der einen Seite mit der Salzzusammensetzung 
des Meerwassers auf der andern Seite harmonisiert; damit gewinnt die Bewegungsperiodik, die für unseren Organismus Existenzbedingung ist, biologisch einen Zusammenhang mit den Lebenserscheinungen primitivster einzelliger Organismen des Meerwassers, indem die inneren Bedingungen noch den deutlich erhaltenen Zusammenhang mit äußeren Bedingungen zeigen, die stammesgeschichtlich auBerordentlich früh wirksam waren und später ihre Wirksamkeit als äußere Bedingungen verloren.

\section{Celluläre Periodik.}

Charakteristisch für eine Periode ist: 1 . die Dauer der Periode, 2. die Amplitude, 3. die Form des Zustandwechsels. Danach stellt die Teilung einer befruchteten Eizelle in 2, dann in 4 Zellen usw. einen periodischen Vorgang vor, wobei nach der Teilung der frühere Zustand der Zelle samt dem Kern, strukturell wieder erreicht wird, die Dauer der Periode durch die Dauer des Teilungsvorganges der Zelle ausgedrückt und die Amplitude durch die gesamten strukturellen Aenderungen im Kern und Protoplasma im Teilungsakte dargestellt wird. Wie man für jedes Chromosom die Ortsveränderung als Marke in ein Ordinatensystem eintragen könnte und somit eine Pendelung nachweisen kann, so kann man es für jeden Punkt der gesamten sich teilenden Zelle + Kern. Es ist nicht unsere Aufgabe, etwa energetisch hier diesen Akt der Zellteilung näher betrachten zu wollen, sondern es kommt uns lediglich auf die Festlegung der Tatsache an, daß die Zellteilung e in periodischer Vorgang ist. Da aber jede Bildung des Organismus nur durch Zellteilung zustande kommen kann, so ist das Werden des Organismus in summa eine Periodik. Diese Tatsache ist jedenfalls fundamental. Verfolgt man etwa an einem befruchteten Seeigelei die Zeiten der Zellteilung und die Modalitäten der Zellteilung, so würde sofern die Zellteilung progressiv nach der Formel, $2,2^{2}, 2^{3}, 2^{4}, 2^{5} \ldots 2^{\text {n }}$ vor sich ginge und die Zellteilung eine zeitlich gleichmäßige wäre, die biologische Teilungsperiodendauer der Zelle durch die Gleichung $P=\frac{t}{2^{\text {n }}}$ ausgedrückt worden, wobei $t$ die Zeit und $n$ die Zahl der Teilungsvorgänge darstellte; ja selbst eine etwa zeitlich nicht ganz gleichmäBig verlaufende Zellteilung würde der Formel immer noch den 
Wert einer mittleren Teilungsperiodendauer geben. Nun liegen allerdings die Verhältnisse der Zellteilung komplizierter! Faßt man selbst nur die äquale Furchung ins Auge, wie sie sich bei Säugetieren findet, so ist ein differenzierter formbildender Entwicklungsprozeß überhaupt nur möglich, wenn sich die Zellteilung nicht in jener einfachen Progression weiter fortsetzt, sondern wenn das P r i n zi p des ungleichen Wachstums (0skar Hertwig) mit seinen Flächen, Faltenbildungen nnd Einstülpungen eintritt, indem örtlich determinierte Zellgruppen einen intensiveren Teilungsimpuls erfahren als etwa das Gros der anderen. Es beruht also letzten Endes die Entwicklung überhaupt vom Morulastadium ab auf einer differenten Teilungsperiodik. D a $\mathrm{m}$ it e $\mathrm{n} \mathrm{t} f \mathrm{a} l \mathrm{llt}$-j e gliche Möglichkeit die Periodendauer der Zellteilung wachsender Individuen einheitich $z u$ beurteilen. Die Gleichung $P=\frac{t}{2^{n}}$ trifft also für den wachsenden und sich geweblich differenzierenden Organismus nicht zu, die Zahl der gebildeten Zellen ist vielmehr eine Interferenzerscheinung aus vielen zeitlich verschieden dauernden Perioden der Zellteilung. Eine derartig unübersehbare Periodik hat nur für uns den Wert eines Gedankenexperimentes, wir schließen daraus, da $B$ für den wachsenden Organismus die $Z$ urückführung einer Periodik der Lebensvorgänge auf eine Periodik der sich teilenden Zellen wegen der Unübersehbarkeit der verschiedenen Zellteilungen ein Ding der Unmöglichkeit ist. Dagegen müssen wir in dem Wachstum eine $\mathrm{S}$ u $\mathrm{m} \mathrm{m}$ e periodischer Vorgänge der Zellteilungen sehen; der Begriff der Wachstumsgeschwindigkeit kann wohl den Begriff dermittleren Periodeda a r der Zellteilung des wachsenden Indivi du u m a blösen. Ersetzt man die Wachstumsgeschwindigkeit durch die prozentische Zunahme der Körpersubstanz, ausgedrückt als Masse durch das Grammgewicht und als Funktion der Zeit (als Zeiteinheit der 24stündige Tag), so kann man eine Kurve für diese Wachstumsgeschwindigkeit von dem ersten Beginn der Eiteilung bis zum Absterben für jedes Individuum gewinnen. Für den Menschen hat z.B. Friede $\mathrm{n}$ th a 1 solche Kurven aufgestellt. Dieser Autor hat auch vergleichende Untersuchungen über die 
Wachstumsgeschwindigkeit bei Säugetieren angestellt und gefunden, daß die prozentische Zunahmegeschwindigkeit der Säugetiere von den ersten Lebensstadien mit geringen Schwankungen durch die ganze Wachstumsperiode absinkt und daß gleich große Tiere aus ganz verschiedenen Säugeordnungen annähernd gleich rasch wachsen, wenn man gleiche Altersstufen vergleicht, daß sie dagegen ungleich rasch wachsen, wenn man die Neugebornen vergleicht. Die Wachstumsgeschwindigkeit beim Menschen hat übrigens eine große Aehnlichkeit mit der Reaktionsgeschwindigkeitskurve zweier in Reaktion tretender chemischer Substanzen, wobei die Kurve um so steiler zu Beginn verläuft, je stärker die Konzentrationen sind. Diese Dinge sind ja aus dem Massenwirkungsgesetz von $\mathrm{G} u \mathrm{l} \mathrm{d}$ b e $\mathrm{rg}$ und W a a g e geläufig. Beim Menschen erreicht nun die Wachstumsgeschwindigkeit vom 25-50 Jahre fast einen Nullwert, im 6 . und 7 . Dezennium nimmt sie sogar einen negativen Wert an. Die Tatsache, daB die Wachstumsgeschwindigkeit mit dem 25. Jahre fast den Nullwert erreicht, läßt sich auch so ausdrücken: di e W a chstumsperiodik strebt einem Gleichgewichts$z$ ust and $z$ u. Obwohl die Körpermassenzunahme ja nicht nur durch Zellen repräsentiert wird, sondern auch die Interzellularsubstanzen eine große Rolle spielen ferner auch totes aufgespeichertes Nährmaterial, so darf man doch auf Grund unserer bisherigen biologischen Kenntnisse sagen, daß dieser Satz im allgemeinen durchaus berechtigt ist. Die Zahl der Ganglienzellen und Herzmuskelzellen, die Zahl der Muskelelemente ist schon bei der Geburt festgelegt. Die parenchymatischen Organe vermehren sich noch, bis das Individuum in seinem Wachstum abgeschlossen ist. Nur das Blut, Haut, Haare und Nägel zeigen eine Periodik des zellulären Wachstums, wobei bei den roten Zellen des Blutes selbst die Gleichung $\frac{D}{A}=1$ zutrifft, d. h. Blutzerfall und Regeneration beschreiben einen KreisprozeB. Also auch hier trotz der rhythmischen Zellneubildung das Auftreten eines Gleichgewichtszustandes. Nur bei Haut, Haaren und Nägel ist davon nicht zu reden. Die Tatsache, daß eine zelluläre Periodik nach Abnahme derWachstumsgeschwindigkeit auf den niedrigsten Wert, den wir einmal den Nullwert nennen wollen, kaum noch existiert, berechtigt uns zu der Ansicht, daß man nicht von einer Periodik der Lebensvorgänge im allgemeinen reden darf, denn schließlich ist, wenn von Werden und Vergehen der, Sub- 
stanzen" d. h. Elemente unseres Körpers geredet wird, doch immer die Zelle das werdende bzw. vergehende Element. Man könnte nun, um sich überhaupt eine Vorstellung zu machen, wie groß die zelluläre Periodik beim ausgewachsenen Individuum ist, den Versuch machen, die Zahl der täglich sich neubildenden Epidermiselemente, Haarelemente und Hornelemente etwa dadurch zu bestimmen, daß man den Nukleingehalt dieser durch den Purinbahnwert bestimmt; alsdann käme man aber auf auBerordentlich kleine Werte pro 24 Stunden, zumal da der Stickstoffwert aller dieser Elemente ein außerordentlich kleiner ist. Größere Ausschläge erhielte man, wenn man den Wert des täglich zugrunde gehenden Blutes bestimmt, was dadurch ermöglicht werden könnte, daB man aus dem im Organismus täglich gebildeten Bilirubin auf die Menge des täglich zerfallenden Hämoglobins schließt. Praktisch ist das allerdings nur. beim Gallenfistelträger möglich. Man kommt hier auf die Beobachtung der Lebensdauer eines roten Blutkörperchens von 14 Tagen bis 4 Wochen. Für das normale menschliche Individuum ist aber zur Bestimmung dieses Unterganges der roten Blutelemente keine Möglichkeit gegeben. Dagegen ist eine andere Frage zu diskutieren, ob nämlich aus dem sog. endogenen Harnsäurewert Schlüsse über den Umfang des täglichen Kernunterganges und. der täglichen Kernneubildung erlaubt sind. Bekanntlich scheidet ein normaler Mann bei sog. purinfreier, d. h. praktisch fleischfreier Kost, täglich $0,4-0,6 \mathrm{~g}$ Harnsäure aus, was einem gleichen Werte von umgesetzten Purinbasen aus seinen Kernnukleinen entspricht. Bedenkt man, daß man aus $100 \mathrm{~g}$ Muskulatur etwa $0,1 \mathrm{~g}$ der Purinbasen darstellen kann, aus $100 \mathrm{~g}$ Leber etwa $0,3 \mathrm{~g}$, so könnte man zu der Meinung kommen, daß der Wert des zugrunde gegangenen Kernmateriales innerhalb 24 Stunden bei einem Menschen ein außerordentlich großer sei, demgegenüber der Zerfall von rotem Blute oder der Abstoßung von Horn, Haut, Haaren ein verschwindend kleiner ist. Indessen kommt man doch in dieser Frage zu einer anderen Auffassung, wenn man die Verhältnisse des absoluten Hungers oder der chronischen Unterernährung ins Auge faßt. Es sinkt unter diesen Verhältnissen nämlich keineswegs der Harnsäurewert, dabei kann der Gesamteiweißumsatz auf 20-30 g Eiweiß herabgesetzt sein. Es wäre also dann der Nuklein- d. h. Kernumsatz größer als der Protoplasmaumsatz, was nicht gut denkbar ist. So kommt man $z u$ der Ansicht, daB dieser endogene Purinbasenumsatz weiter nichts wie 
ein Auswechsein beschädigter Teile ist, die ohne den Kern in seiner Integrität zu beeinflussen auf synthetischem Wege wieder ersetzt werden. Immerhin ist die Menge dieser auswechselbaren Nukleinteile eine relativ große. Wir können aber nicht von periodischem Kernuntergang und Neubildung sprechen, vielmehr nur von einem stationären Prozesse im Sinne $\frac{\mathrm{D}}{\mathrm{A}}=1$.

Wenden wir uns jetzt einer Kardinalfrage zu. Warum nimmt die Wachstumsgeschwindigkeit eines Organismus mit der Lebensdauer $a b$ ? Nennen wir $P$ die Teilungspotenz einer Zelle, so würde bei abnehmender Teilungspotenz einer Zelle das Symbol $P_{1}>P_{2}>P_{3}>$ $P_{4} \ldots P_{n-1}>P_{n}$ und $P_{n}=0$ ausdrücken, daß an einer bestimmten Teilungsperiodik diese Teilungspotenz erloschen ist. Die befruchtete Eizelle hat jedenfalls den Wert von $P_{1}$ und für die Ganglienzellen und Muskelzellen kann man schon sehr früh d. h. nach ihrer Differenzierung den Wert von 0 feststellen, d. h. mit ihrer Anlage erlischt die Möglichkeit einer Neubildung durch Zellteilung. Aber auch bei den Parenchymzellen unserer inneren Organe wie Leber und Niere scheint doch die Periodik begrenzt zu sein, wenigstens unter normalen Bedingungen, während andere Organe sich eine Periodik bewahren. Bezüglich der Geschlèchtszellen ist jedenfalls die Periodik auch eine begrenzte; so wird nach $\mathrm{W}$ a I d e y e $r$ keine Eizelle mehr im Ovar nach dem 2. Lebensjahre beim Menschen neu gebildet. Andrerseits zeigen andere Epithelzellen z. B. die Zellen der Schleimhäute wie der Epidermis eine in gewissem Sinne unbegrenzte Periodik. Das obenangeführte Symbol für die Abnahme der Teilungspotenz der Zellen ist also nicht vollgültig für den menschlichen Organismus, da es in der Teilungsperiodik Reihen gibt, in denen das Wort $P_{n}=0$ und andrerseits der Wert $\infty$ ist, dabei überwiegen allerdings die begrenzten Teilungspotenzen derart die unbegrenzten, daß praktisch eine Abnahme der Wachstumsgeschwindigkeit auf den Wert 0 nach der Pubertät eintritt, ein Wert, der allerdings wieder nur erreicht werden kann durch die stationäre Periodik einer Anzahl von zugrundegehenden und werdenden Zellelementen im obengenannten Sinn von $\frac{D}{A}=1$. Vergleicht man das Wachstum der Säugetiere, Vögel, Reptilien usw., das sich bei einer bestimmten Größe des Individuums begrenzt mit dem Wachstum, wie man es sonst in der 
belebten Natur findet, so findet man einen Gegensatz zu Wachstum der Bäume, Sträucher, Muscheln, Cephalopoden ja selbst bei vielen Fischen, indem hier das Wachstum nach der Geschlechtsreife ruhig weiter geht: es ist, auch wenn es mit zunehmender Größe des Organismus abnimmt, nicht begrenzt. Noch schärfer ist der Gegensatz zur Karzinomzelle, die ein nur durch Nährboden begrenztes Wachstum zeigt. Warum hört aber bei den Säugetieren das Wachstum auf, d. h. nimmt die Teilungsperiodik der den Organismus aufbauenden Elemente zu einem bestimmten Zeitpunkte bis auf 0 ab? Wir können die Erklärung nur in der Organisation des Säugetierorganismus sehen und zwar speziell in einer Differenzierung, durch die mit zunehmender Differenzierung das Wachstum hormonal reguliert wird, indem bestimmte Drüsenkomplexe (innere Drüsen: Hypophyse, Thymus, Schilddrüse, Epiphyse u. a.) eine Wachstumsbestimmende hormonale Drüsenfunktion übernehmen, wodurch die zelluläre Wachstumsperiodik ihrer Automalie entkleidet und anderen höheren Instanzen unterworfen wird. So wird letzten Endes die Wachstumsperiodik zu einer hormonalen Periodik, mit der wir uns nunmehr zu befassen haben. Nur ein Zellsystem existiert, das sich dem Einflusse hormonaler Periodik entzieht und dabei die volle Potenz zur rhythmischen Teilung mit allen Möglichkeiten der Differenzierung wahrt, das ist die Geschlechtszelle; a $\mathrm{n}$ di eser beginnt die zelluläré Rhythmik von neuem.

\section{Hormonale Periodik.}

Die Menstruationsperiodik ist die deutlichste Rhythmik einer hormonalen Periodik, die an eine bestimmte innere Drüse, die Eierstöcke gebunden ist und die mit hormonalbedingten Allgemeinund Lokalerscheinungen einhergehen. Daß diese Rhythmik mit der Funktion der Eierstöcke zusammenhängt, läßt sich ja einfach dadurch beweisen, daß sie verschwindet, sobald die Eierstöcke exstirpiert sind. Bei der menschlichen Frau ist die häufigste Dauer der Menstruationsperiodik das Intervall von 28 Tagen (,Menses“), was manche Autoren dazu veranlaßt, dieses Intervall auf den Einfluß des Mondes zurückzuführen. So wenig der Einfluß der Jahreszeiten auf den Organismus unterschätzt werden kann, und wir werden zeigen, daß solche hormonalen Einflüsse bestehen müssen, so sind wir doch andrerseits nicht in der Lage, die Abhängigkeit der 
Periode der menschlichen Frau vom Monde anzuerkennen, denn abgesehen davon, daß alle Frauen zu gleicher Zeit menstruieren müßten, so würde sich die Menstruationsperiodik der Frau wesentlich biologisch von der Menstruationsperiodik anderer Tiere unterscheiden, bei denen sicherlich eine Mondperiodik nicht vorhanden ist. Wie die ovariogene Menstruationsrhythmik zustande kommt, ist nicht zu erklären, sicher ist aber ihr keine sexuelle Periodik, ausgehend von den Geschlechtsdrüsen des Mannes, an die Seite $z u$ stellen; auch die Libido des Mannes kennt keine starre Periodik, sie ist von äußeren Bedingungen wesentlich abhängig.

Weniger deutlich als die ovariogene Menstruationsperiodik zeigt sich die Periodik der übrigen Drüsen mit innerer Sekretion. Man kann nur das gesteigerte Wachstum zur Zeit der Pubertät als greifbaren Ausdruck gesteigerter hormonaler Wirkung auf die zelluläre Periodik ansehen, vor a $11 \mathrm{e} \mathrm{m}$ a ber d a s $\mathrm{ung} l \mathrm{e}$ i c hmäBige Wachstum zubestim m ten Jahreszeiten be i $\mathrm{Kinde} \mathrm{r}$ und in bestimmten Entwicklungsperioden.

\begin{tabular}{|c|c|c|c|c|c|c|c|}
\hline Periode & Wachstum & & Knaben & & & Mädchen & \\
\hline 1 & rasches & bis zum & 5. -6. & Jahr & bis & $\operatorname{zum} 5 .-6$ & Jahr \\
\hline 2 & langsames &,,, & $10 .-12$ & ", & $"^{\cdot}$ &,$\quad 10$ & $"$ \\
\hline 3 & beschleunigtes & , & 16. - 18. & " & $"$ & , 14.-15. & \\
\hline 4 & verlangsamtes & , & 25. & & , &,$\quad 18 .-20$ & \\
\hline
\end{tabular}

Dieser Wachstumsrhythmus, der mit hormonalen innersekretorischen Verhältnissen unverkennbar verknüpft ist, findet sich durchgehends bei der europäischen Rasse. Und von der Jahresperiodizität des Wachstums berichtet $M$ a r t in (Lehrbuch der Anthropologie 1914, Gustav Fischer Jena), daß diese von den allgemein klimatischen Bedingungen abzuhängen scheint und daher auch in nordischen Ländern, wo der klimatische Wechsel schroffer ist, deutlicher ausgeprägt ist. ,,Sie besteht darin, daß die stärkste Längenzunahme auf die erste Jahreshälfte (Februar-August), besonders aber auf die Monate Juli-August fällt, und daß in der 2. Jahreshälfte (September-Januar) nur ein geringes Wachstum eintritt (S c h m i d-M o m e a r d)."

Wenn wir also das Wachstum in der zellulären Periodik wie in seiner Gesamtheit daraufhin prüfen, inwieweit sich mit äußeren periodischen Faktoren der Umwelt Beziehungen finden, so läßt sich für den Menschen nur die eine Tatsache feststellen, daß die 
Jahreszeiten einen solchen Einfluß beim wachsenden Individuum ausüben. Für die zelluläre Periodik lassen sich im einzelnen keine solche Beziehungen auffinden, es sei denn daß z. B. das stärkere Wachstum der Haare, vielleicht auch stärkerer Untergang von Gewebe (Blut, Schleimhautepithel, Epidermis) im Wachzustande gegenüber dem Schlafe als eine solche periodische Abhängigkeit angesehen werden sollte, das sind indessen Erscheinungen, wie sie samt und sonders unter den Begriff der Schlafperiodik zusammengefaßt werden können, in dem die Aktivität der Lebenserscheinungen, wie noch auseinandergesetzt werden soll, gegenüber dem Wachzustande herabgesetzt ist.

\section{Die Periodik des Schlafes.}

Hier wie nirgends zeigt sich sinnfällig der unmittelbare Einfluß der umgebenden Natur auf unseren Organismus. Tag und Nacht, Wachen und Schlaf sind fest verknüpfte Beziehungen, gegen die auch der Kulturmensch, der sich von den Beziehungen zur Natur nach Möglichkeit frei macht, auf die Dauer nicht ungestraft, angehen kann. Durchgehends charakterisiert den Schlaf nicht nur die Ausschaltung des Oberbewußtseins, sondern wie schon gesagt die Erniedrigung sämtlicher unserem wissenschaftlichen Erfassen zugänglichen Lebensäußerungen: der Stoffwechsel sinkt, der Blutdruck sinkt, Puls- und Atmungsfrequenz nehmen ab usw. Im stationären Prozesse ist der Schlaf die Lebenslage, in der D den geringsten Wert bekommt, daher ein Regulativ zur Aufrechterhaltung der Gleichgewichtslage. Im allgemeinen reiht sich der Schlaf biologisch in die Gruppe der Lebenserscheinungen, die man zwar nicht als Inaktivitätserscheinungen schlechtweg bezeichnen kann, die sich aber durchgehends als Einschränkung der Lebenstätigkeit charakterisieren. Solche Zustände findet man bekanntermaßen in der Botanik sehr ausgedehnt, aber auch im Tierreiche. Der Winterschlaf von Säugern ist der deutlichste Ausdruck an eine Anpassung ökologisch ungünstiger Verhältnisse. Beim Schlaf des Menschen tritt das ökologisch ungünstige der Lage nicht primär hervor, wenngleich z. B. auf Irland die Fischer im Winter einen Schlaf schlafen, der sich dem Winterschlaf der Tiere sehr nähert. Der natürliche Schlaf unterscheidet sich allerdings quantitativ und qualitativ wesentlich vom Winterschlaf der Tiere. Auf diese Dinge soll hier nicht 
näher eingegangen werden, so verlockend es auch wäre, die Theorie des Schlafes hier abzuhandeln. Die eine Tatsache läßt sich aber als zweifellos hinstellen, daß die fehlende Sonne als Licht- und Wärmespenderin es ist, die den Schlaf als veränderte Lebenslage herbeiführt, so daß der Schlaf eine Anpassungserscheinung an die Sonnenperiodizität genannt werden darf.

So wollen wir die Aufzählung der periodischen Erscheinungen unseres Organismus in somatischer Beziehung beschließen und uns davon fernhalten, die Periodizität psychischen Geschehens mit in die Diskussion hineinzuziehen. Der mit Intellekt begabte zu metaphysischen Spekulationen nur allzu geneigte Mensch verfällt, wenn er die psychische Periodizität abhängig sein lassen will von Faktoren der Außenwelt, in das Gebiet der Mystik. Davon wollen wir uns freihalten. Das Kapitel der Periodizitäten mag einen psychophysischen Zusammenhang höchstens bei Störungen der inneren Drüsentätigkeit ergeben und auch hier nur beschränkt auf das Affektleben der Frau. Darüber hinaus aber, und selbst da steht man auf schwankendem Boden. Der Mensch möchte, nicht zuletzt auf psychischem Gebiet, Verknüpfungen mit den kosmischen Periodizitäten annehmen. Ihm genügt es nicht, wenn eine bestimmte somatische Periodizität schwächere oder stärkere Beziehungen mit der Umwelt hat: er möchte am: liebsten selbst mit tausend Fäden am Kosmos hängen und die mystische Astrologie am liebsten zu neuem Dasein erwecken; spekulative Hypothesen von F1i e B z. B. sind ganz abgesehen davon, daB psychisches und somatisches Geschehen gleichgestellt sind, wissenschaftlich nicht überprüfbar, da eine methodische Grundlage, die Voraussetzung jeder Wissenschaft fehlt und eine Hypothese zwar wissenschaftliche Beobachtungen überbrücken kann, aber niemals selbst das Fundament der Wissenschaft bilden kann. Die Gesetzmäßigkeiten von Werden und Vergehen im Einzelfalle sind heute zahlenmäßig leicht zurückführbar auf Periodizitäten der Zelle, sowenig beim Menschen wie beim Tiere. Wo somatische Zusammenhänge periodischen Charakters vorhanden sind, da tragen diese Zusammenhänge auch nur ganz allgemein beurteilt den Charakter der Ausgleichserscheinungen an äußere Bedingungen aber nicht etwa den Charakter der Bewahrung ,kosmischer Periodizitäten“. Daß jedes zeitlich-räumliche 
Geschehen nicht kontinuierlich fließt, sondern periodisch kreist, ist dabei durchgehendes Naturgesetz, oder sagen wir Zweckmäßigkeitsgesetz der Materie, dem sich auch der erfinderische Geist der Menschen anpassen muß: Dampfmaschine, Motor, Turbine, sie zeigen wie überall eine konstante Bewegung in die periodische Bewegung aus Zweckmäßigkeitsgründen gezwängt wird, aber so wenig der goldene Schnitt die Harmonie des Weltalles erfüllen konnte, so unberechtigt wäre es mit der Periodizität des Kosmos schlechtweg den menschlichen Organismus zu erfüllen. 